\title{
Elevated levels of miR-146a and miR-155 in kidney biopsy and urine from patients with IgA nephropathy
}

\author{
Gang Wanga,c, Bonnie Ching-Ha Kwan ${ }^{\mathrm{a}}$, Fernand Mac-Moune Lai ${ }^{\mathrm{b}}$, Kai-Ming Chow ${ }^{\mathrm{a}}$, \\ Philip Kam-Tao Li ${ }^{\mathrm{a}}$ and Cheuk-Chun Szeto ${ }^{\mathrm{a}, *}$ \\ ${ }^{a}$ Department of Medicine and Therapeutics, The Chinese University of Hong Kong, Shatin, Hong Kong, China \\ ${ }^{\mathrm{b}}$ Department of Anatomical and Cellular Pathology, Prince of Wales Hospital, The Chinese University of Hong \\ Kong, Shatin, Hong Kong, China \\ ${ }^{\mathrm{c}}$ Division of Nephrology, Renmin Hospital of Wuhan University, China
}

\begin{abstract}
Background: Previous studies suggested miR-146a and miR-155 play important roles in innate and adaptive immune responses. We studied intra-renal and urinary levels of miR-146a and miR-155 in patients with immunoglobulin A nephropathy (IgAN).

Methods: Intra-renal and urinary levels of miR-146a and miR-155 are quantified in 43 patients with IgAN; the result was compared to 20 nephrectomy specimens and urine sediment of 13 healthy volunteers.

Results: The levels of intra-renal and urinary levels of miR-146a and miR-155 of IgAN are significantly higher than controls. Estimated glomerular filtration rate inversely correlates with intra-renal level of miR-146a and miR-155; proteinuria positively correlates with intra-renal level of miR-146a and miR-155, as well as urinary level of miR-146a and miR-155. Intra-renal level of miR-155 significantly correlates with tubulointerstitial scarring. Urinary level of miR-146a inversely correlates with urinary expression of interleukin (IL)-1 $\beta$, IL- 6 and tumor necrosis factor (TNF)- $\alpha$ and positively correlates with urinary expression of regulated upon activation, normal T-cell expressed, and secreted (RANTES). Urinary level of miR-155 inversely correlates with urinary expression of IL- $1 \beta$ and TNF- $\alpha$ and positively correlates with urinary expression of forkhead box P3 (FOXP3) and RANTES.

Conclusion: We conclude that intra-renal and urinary levels of miR-146a and miR-155 were significantly elevated in IgAN, and the degree of upregulation correlates with clinical and histological severity of the disease. Our results suggested miR-146a and miR-155 might play an important role in the pathophysiology of IgAN.
\end{abstract}

Keywords: microRNA, proteinuria, chronic renal failure

\section{Introduction}

Immunoglobulin A nephropathy (IgAN) is the most common form of primary glomerulonephritis worldwide [1]. IgAN is also an important cause of endstage renal disease (ESRD); 15 to $40 \%$ of patients with

${ }^{*}$ Corresponding author: Dr. C.-C. Szeto, Department of Medicine and Therapeutics, Prince of Wales Hospital, The Chinese University of Hong Kong, Shatin, NT, Hong Kong, China. Tel.: +86 8522632 3126; Fax: +86 8522637 3852; E-mail: ccszeto@cuhk.edu.hk. biopsy-proven $\operatorname{IgAN}$ progress to ESRD in 10 to 20 years $[2,3]$.

Although the exact etiology of $\operatorname{IgAN}$ is not fully elucidated, mucosal and systemic immune responses have been suggested to play a central role in the pathogenesis of IgAN [4,5]. Disorder of B and T lymphocytes has been described throughout the clinical course of IgAN [6,7]. However, the underlying mechanisms of immune response activation in IgAN have not been completely clarified.

MicroRNAs (miRNAs) are noncoding, single-stranded RNA molecules of 21 to 23 nucleotides in length; 
miRNAs regulate gene expression at post-transcriptional level by degrading or blocking translation of messenger RNA (mRNA), and play important roles in many physiological and pathological processes [8-11]. A number of miRNA species, notably miR-146a and miR-155, have been shown to regulate multiple steps in the development and function of lymphocytes and myeloid cell $[12,13]$.

The role of miRNA in the pathogenesis of IgAN remains unknown. A previous study that used miRNA microarray technology revealed intra-renal dysregulation of a number of miRNA species in patients with IgAN [14]. Our previous studies showed that intrarenal levels of miRNA related to epithelial-mesenchymal transition (EMT), such as miR-200c, miR-141, miR-205 and miR-192, were diversely regulated and correlated with disease severity and progression in patients with IgAN [15], while urinary level of EMTrelated miRNA such as miR-200a, miR-200b and miR429 , were reduced, and the degree of reduction correlated with disease severity and rate of progression [16]. In the present study, we examined the intra-renal and urinary levels of two immune-related miRNAs, miR$146 \mathrm{a}$ and miR-155, in patients with $\operatorname{IgAN}$.

\section{Patients and methods}

\subsection{Subjects}

We studied 43 consecutive patients with IgAN confirmed by kidney biopsy in the Prince of Wales Hospital, Hong Kong, between 2004 and 2007. Patients with other coexisting renal pathology, and recurrent IgAN after kidney transplantation, were excluded. The study was approved by the Clinical Research Ethical Committee of the Chinese University of Hong Kong. All patients provided informed consent. Clinical data including serum creatinine and 24 hours urine protein were recorded at the time of kidney biopsy. Glomerular filtration rate (GFR) was estimated by a standard equation [17]. We studied normal renal tissue from the nephrectomy specimen of 20 patients with renal cell carcinoma (all clear cell type) and urine sediment from 13 healthy subjects as controls for intra-renal and urinary expression study, respectively.

After renal biopsy, all patients were followed every 2 months for at least 12 months. Renal function was assessed at every visit. Disease progression was measured by the rate of GFR decline, which was calculated by the least-square regression method [18]. Treat- ment for individual patient was determined by the responsible physician and not affected by this study. All physicians were blinded from the results of miRNA and mRNA expression.

\subsection{Sample preparation}

Immediately after kidney biopsy, the renal tissue was fixed in $10 \%$ neutral buffered formaldehyde overnight and then dehydrated by alcohol and embedded in paraffin for intra-renal miRNA and mRNA expression. Ten $10 \mu \mathrm{m}$ sections were cut from the formalin-fixed and paraffin-embedded (FFPE) tissue blocks using a microtome and pooled in a $1.5 \mathrm{ml}$ microcentrifuge tube. The sections were then treated by xylene for 3 minutes at $50^{\circ} \mathrm{C}$ and washed by $100 \%$ ethanol twice. Air dry the pellet for 30 minutes at room temperature.

Urine specimen was collected and processed immediately. Urine sample was centrifuged at $3000 \mathrm{~g}$ for 30 minutes and at $13000 \mathrm{~g}$ for 5 minutes at $4^{\circ} \mathrm{C}$. Supernatant was discarded and the urinary cell pellet was lysed by RNA lysis buffer (Qiagen Inc, Ontario, Canada). Specimens were then stored at $-80^{\circ} \mathrm{C}$ until use.

\subsection{Measurement of miRNA levels}

RecoverALL ${ }^{\mathrm{TM}}$ total nucleic acid isolation kit and MirVana ${ }^{\mathrm{TM}}$ miRNA isolation kit (Ambion, Inc. Austin, TX, USA) were used for the extraction of total RNA from FFPE tissue and urinary sediment according to the manufacturer's protocol.

TaqMan ${ }^{\circledR}$ microRNA reverse transcription Kit (Applied Biosystems, Foster City, CA, USA) and High Capacity cDNA Reverse Transcription Kit (Applied Biosystems, Foster City, CA, USA) were used for reverse transcription. For miRNA, $0.5 \mu \mathrm{g}$ total RNA was mixed with $1 \mu \mathrm{l}$ specific primers, $0.05 \mu \mathrm{l} 100 \mathrm{mM}$ dNTPs (with dTTP), $0.5 \mu \mathrm{l} 10 \mathrm{x}$ reverse transcription buffer, $0.33 \mu \mathrm{l}$ (50U) MultiScribe ${ }^{\mathrm{TM}}$ Reverse Transcriptase, $0.66 \mu \mathrm{l}$ RNase inhibitor $(20 \mathrm{U} / \mu \mathrm{l})$ and made up to $5 \mu \mathrm{l}$ with $\mathrm{H}_{2} \mathrm{O}$. Reverse transcription was performed at $16^{\circ} \mathrm{C}$ for 30 minutes, $42^{\circ} \mathrm{C}$ for 30 minutes and $85^{\circ} \mathrm{C}$ for 5 minutes. For mRNA, $3 \mu \mathrm{g}$ total RNA was mixed with $2 \mu \mathrm{l}$ specific primers, $0.8 \mu \mathrm{l} 100 \mathrm{mM}$ dNTPs (with dTTP), $2 \mu \mathrm{l} 10 \mathrm{x}$ reverse transcription buffer, $1 \mu \mathrm{l}$ (50U) MultiScribe ${ }^{\mathrm{TM}}$ Reverse Transcriptase, $1 \mu \mathrm{l}$ RNase inhibitor $(20 \mathrm{U} / \mu \mathrm{l})$ and made up to $20 \mu \mathrm{l}$ with $\mathrm{H}_{2} \mathrm{O}$. Reverse transcription was performed at $25^{\circ} \mathrm{C}$ for 10 minutes, $37^{\circ} \mathrm{C}$ for 120 minutes and $85^{\circ} \mathrm{C}$ for 5 minutes. The resulting cDNA was stored in $-80^{\circ} \mathrm{C}$ until use. 
Table 1

Demographic and baseline clinical data

\begin{tabular}{lccc}
\hline & IgAN & Biopsy control & Healthy subject \\
\hline No. of case & 43 & 20 & 13 \\
Sex (M:F) & $27: 16$ & $13: 7$ & $8: 5$ \\
Age (year) & $48.4 \pm 12.6$ & $52.2 \pm 8.3$ & $31.8 \pm 4.0$ \\
Blood pressure (mmHg) & & & \\
systolic & $141.8 \pm 25.8$ & $127.8 \pm 13.7$ & - \\
diastolic & $81.9 \pm 14.5$ & $81.5 \pm 7.1$ & - \\
Proteinuria (g/day) & $1.13 \pm 1.13$ & $0.01 \pm 0.03$ & 0.00 \\
Serum creatinine $(\mu \mathrm{mol} / \mathrm{l})$ & $175.1 \pm 123.0$ & $95.1 \pm 23.0$ & - \\
GFR $\left(\mathrm{ml} / \mathrm{min} / 1.73 \mathrm{~m}^{2}\right)$ & $50.4 \pm 29.5$ & $76.1 \pm 26.0$ & - \\
\hline
\end{tabular}

IgAN, immunoglobulin A nephropathy; GFR, glomerular filtration rate.
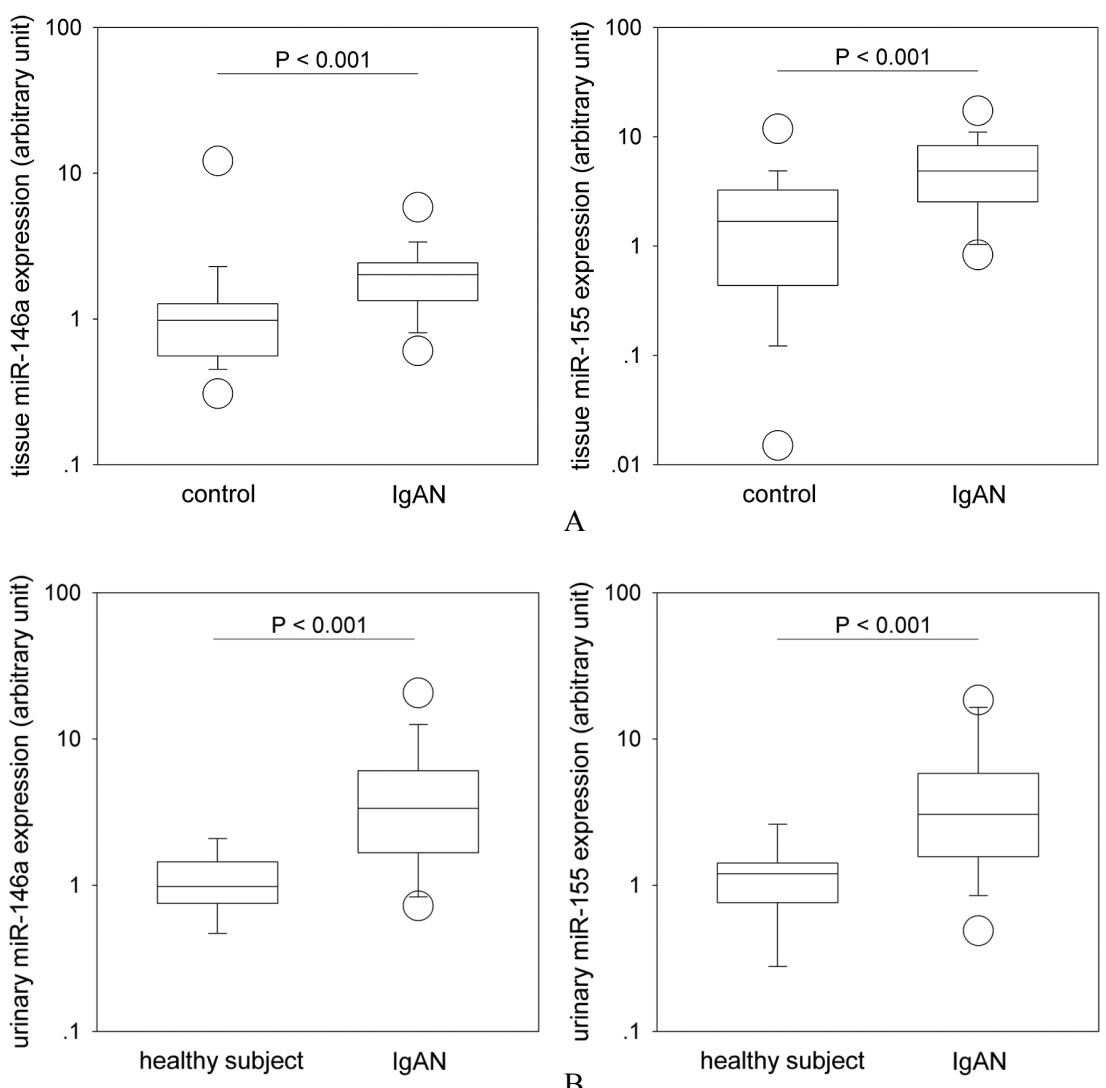

Fig. 1. Comparison of intra-renal (Fig. 1A) and urinary (Fig. 1B) levels of miRNAs between patients with IgAN and normal controls (CTL). Data are compared by Mann-Whitney U test. Levels are represented as ratio to the average of controls.

Intra-renal and urinary levels of miR-146a and miR155 , together with urinary mRNA of inteleukin- $1 \beta$ (IL-1 $\beta$ ), interleukin-6 (IL-6), tumor necrosis factor$\alpha$ (TNF- $\alpha$ ), forkhead box P3 (FOXP3) and Regulated upon Activation, Normal T-cell Expressed, and Secreted (RANTES) were quantified by RT-QPCR using the ABI Prism 7900 Sequence Detection System (Applied Biosystems, Foster City, CA, USA). Commercially available Taqman primers and probes, including
2 unlabeled PCR primers and 1 FAM $^{\mathrm{TM}}$ dye-labeled TaqMan ${ }^{\circledR}$ MGB probe were used for all the targets (all from Applied Biosystems). For mRNA expression, the primer and probe set was deliberately designed across the intron-exon boundary so as not to detect probable genomic DNA. For RT-QPCR, $2.5 \mu \mathrm{l}$ universal master mix, $0.25 \mu \mathrm{l}$ primer and probe set, $0.33 \mu \mathrm{l}$ cDNA and $1.92 \mu \mathrm{l} \mathrm{H}_{2} \mathrm{O}$ were mixed to make a $5 \mu \mathrm{l}$ reaction volume. Each sample was run in triplicate. RT-QPCR 

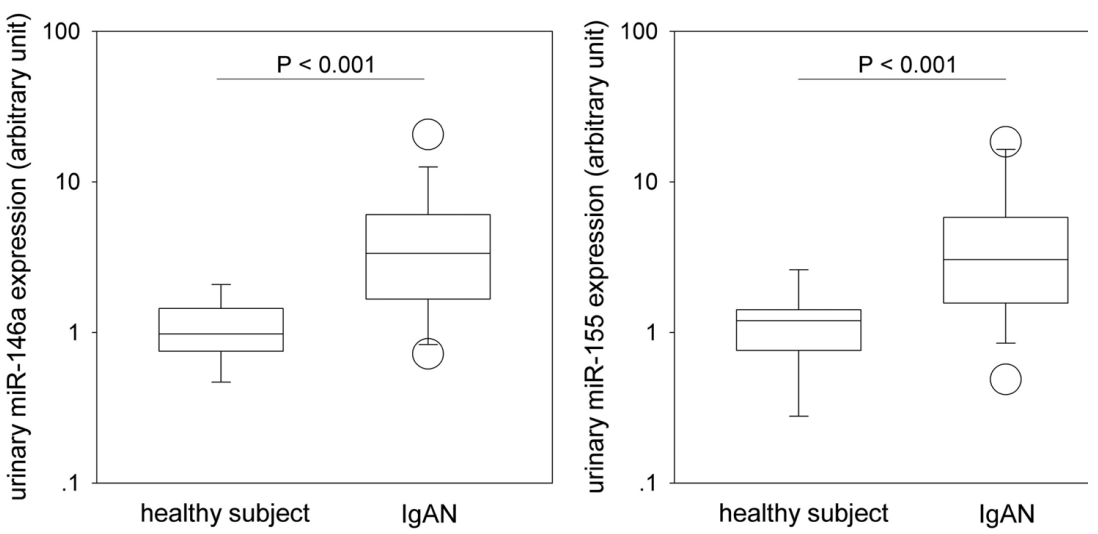

Fig. 2. Relation between intra-renal and urinary levels of miRNAs. Data are compared by Spearman's rank correlation coefficient.

were performed at $50^{\circ} \mathrm{C}$ for 2 minutes, $95^{\circ} \mathrm{C}$ for $10 \mathrm{~min}$ utes, followed by 40 cycles at $95^{\circ} \mathrm{C}$ for 15 seconds and $60^{\circ} \mathrm{C}$ for 1 minute. $\beta$-Glucuronidase (GUSB, Applied Biosystems) and RNU48 (Applied Biosystems) were used as house-keeping genes to normalize the mRNA and miRNA expression respectively $[19,20]$. Results were analyzed with Sequence Detection Software version 2.3 (Applied Biosystems). In order to calculate the differences of expression level for each target among samples, the $\Delta \Delta \mathrm{C}_{T}$ method for relative quantitation was used. Average expression level of normal renal tissue from patients with kidney clear cell cancer and urine from normal subjects was used as calibrator for intra-renal and urinary expression and the expression level of targets was a ratio relative to that of the controls.

\subsection{Assessment of renal scarring}

Analysis of renal fibrosis was determined on $4 \mu \mathrm{m}$ paraffin-embedded sections stained by Periodic Acid Schiff (PAS) or Jones silver stain. The severity of renal fibrosis was scored by an experienced pathologist (FML) who was blinded to the results of molecular studies. The severity of glomerulosclerosis was represented by the percentage of sclerotic glomeruli in total glomeruli obtained from biopsy. For tubulointerstitial scarring, ten microscopic fields were viewed at magnification of $200 \times$ and scored from 0 to $100 \%$ for each patients. The severity of tubulointerstitial scarring was represented by the mean of ten scores.

\subsection{Statistical analysis}

Statistical analysis was performed by SPSS for Windows software version 13.0 (SPSS Inc., Chicago, IL). All the results were presented in mean \pm SD for da- ta normally distributed, and median and inter- quartile range (IQR) for the others. Since data of gene expression levels were highly skewed, either log transformation or nonparametric statistical methods were used. We used Mann-Whitney U test to compare gene expression levels between groups and Spearman's rank-order correlations to test associations between gene expression levels and clinical parameters. When no detectable level of a transcript was found (defined as no detectable level after 40 cycles of RT-QPCR) and there was zero value, a value equal to half of the minimum observed gene expression level was assigned [21]. A P value of below 0.05 was considered statistically significant. All probabilities were two-tailed.

\section{Results}

The demographic and baseline clinical data of the study subjects were summarized in Table 1. As compared to controls, patients with IgA nephropathy had significantly higher level of proteinuria and worse renal function. Histological studies showed that the percentage of glomerulosclerosis and tubulointerstitial scarring were $28.3 \pm 27.0 \%$ and $25.6 \pm 24.1 \%$ respectively.

\subsection{Levels of miRNAs and $m R N A s$}

As compared to control tissue, IgAN had higher intra-renal levels of miR-146a (2.01, IQR 1.33 to 2.43, versus 0.98 , IQR 0.56 to $1.27, P<0.001$ ) and $\mathrm{miR}-155$ (4.85, IQR 2.54 to 8.31 ) versus 1.68 , IQR 0.44 to 3.26 , $P<0.001$ ) (Fig. 1A). Similarly, IgAN group had higher urinary levels of miR-146a (3.35, IQR 1.67 to 6.05 versus 0.98 , IQR 0.75 to $1.44, P<0.001$ ) and miR- 

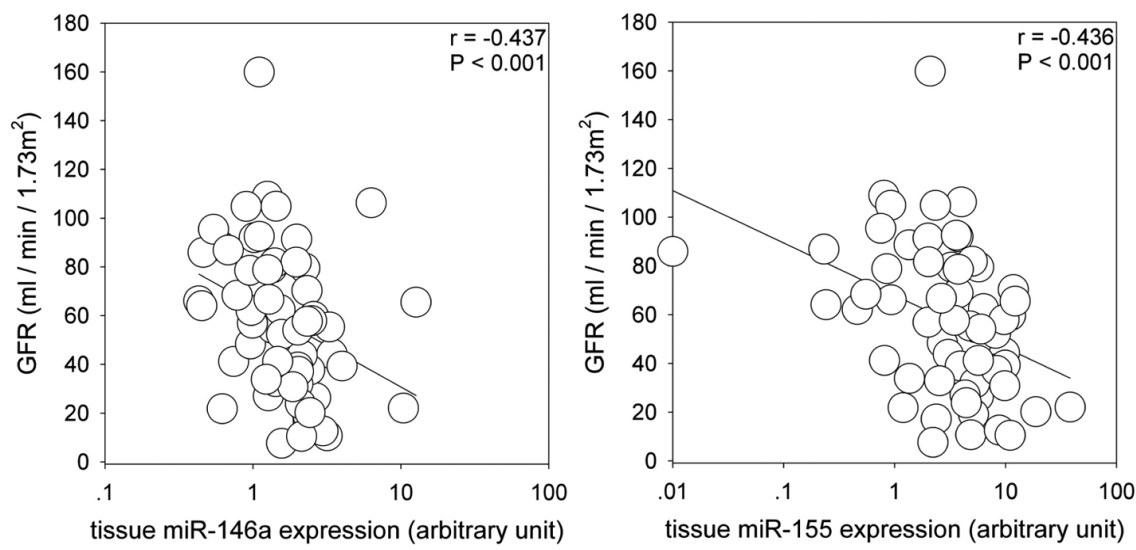

A
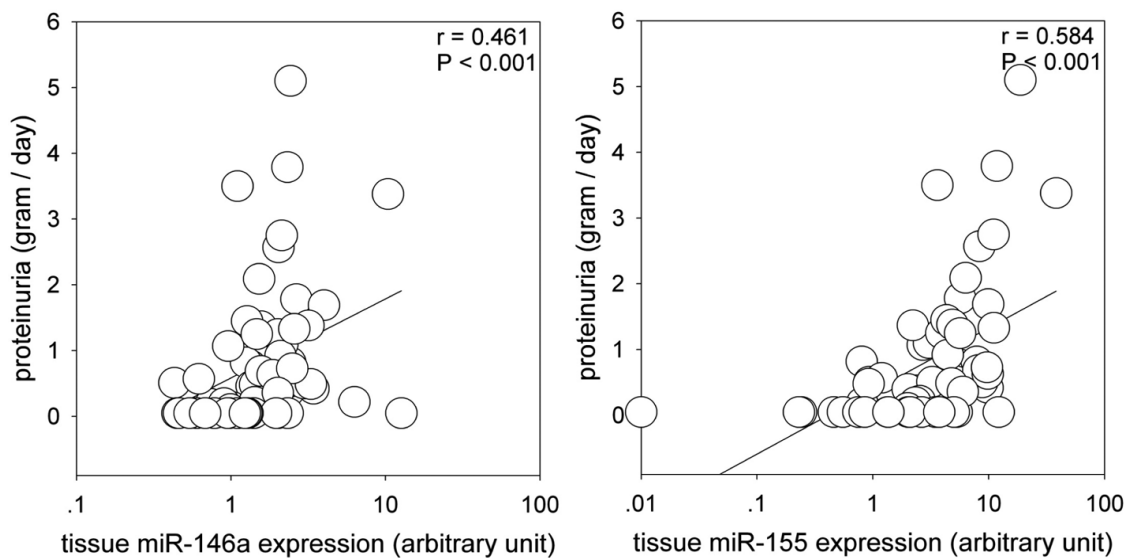

B

Fig. 3. Relation between intra-renal levels of miRNAs and: (A) glomerular filtration rate (GFR); and (B) proteinuria. Data are compared by Spearman's rank correlation coefficient.

155 (3.04, IQR 1.57 to 5.80 versus 1.20 , IQR 0.76 to $1.42, P<0.001$ ) than healthy subjects (Fig. 1B). Further, intra-renal levels of miR-146a and miR-155 had significant internal correlation $(r=0.782, P<0.001)$. Similarly, urinary levels of miR-146a and miR-155 had significant internal correlation $(r=0.809, P<0.001)$ (Fig. 2). There was no significant correlation between tissue and urine level of any of the miRNA studied (details not shown).

\subsection{Relation with clinical and histological parameters}

Intra-renal levels of miR-146a and miR-155 both inversely correlate with GFR and positively correlate with proteinuria (Fig. 3). Urinary levels of both miR$146 \mathrm{a}$ and miR-155 positively correlates with proteinuria (Fig. 4), but neither of them correlates with GFR ( $r=0.074$ and $r=-0.198$, respectively).
Intra-renal level of miR-155 significantly correlates with the degree of tubulointerstitial scarring $(r=0.514$, $P=0.002$ ) (Fig. 5); intra-renal level of miR-146a also correlates with tubulointerstitial scarring $(r=0.322$, $P=0.059$ ), but the result did not reach statistical significance. The degree of glomerulosclerosis, however, do not correlate with intra-renal levels of miR-146a $(r=0.210, P=0.181)$ or miR-155 ( $r=0.205, P=$ 0.193 ). Urinary levels of miR-146a and miR-155 do not correlate with glomerular or tubulointerstitial scarring (details not shown).

\subsection{Relation with urinary cytokine gene expression}

Urinary level of miR-146a inversely correlates with urinary expression of IL- $1 \beta$, IL- 6 , and TNF- $\alpha$, and positively correlates with urinary expression of RANTES (Fig. 6A). Urinary level of miR-155 inversely correlates with urinary expression of IL- $1 \beta$ and TNF- $\alpha$, and 

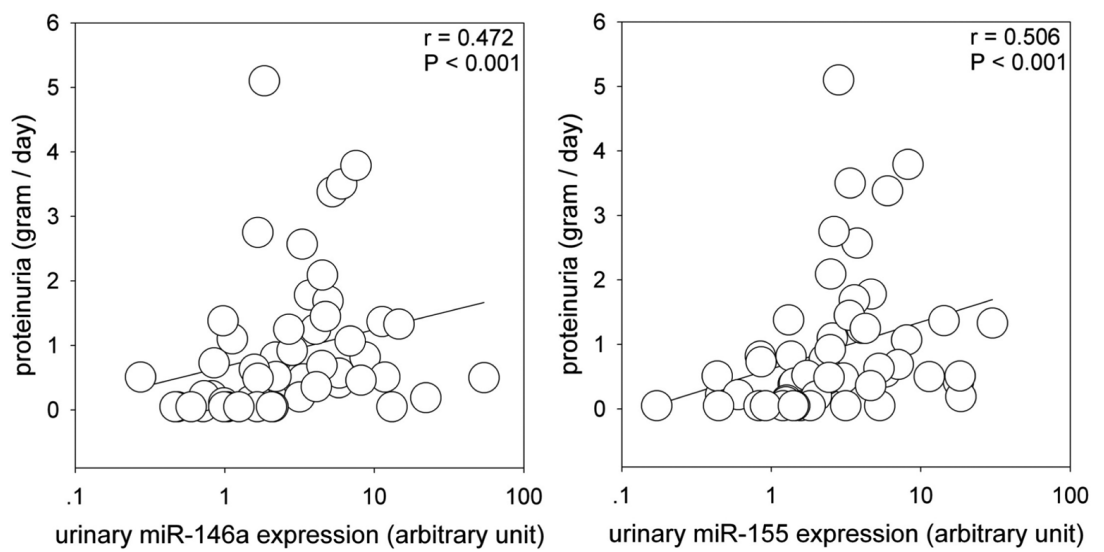

Fig. 4. Relation between urinary level of miRNAs and proteinuria. Data are compared by Spearman's rank correlation coefficient.
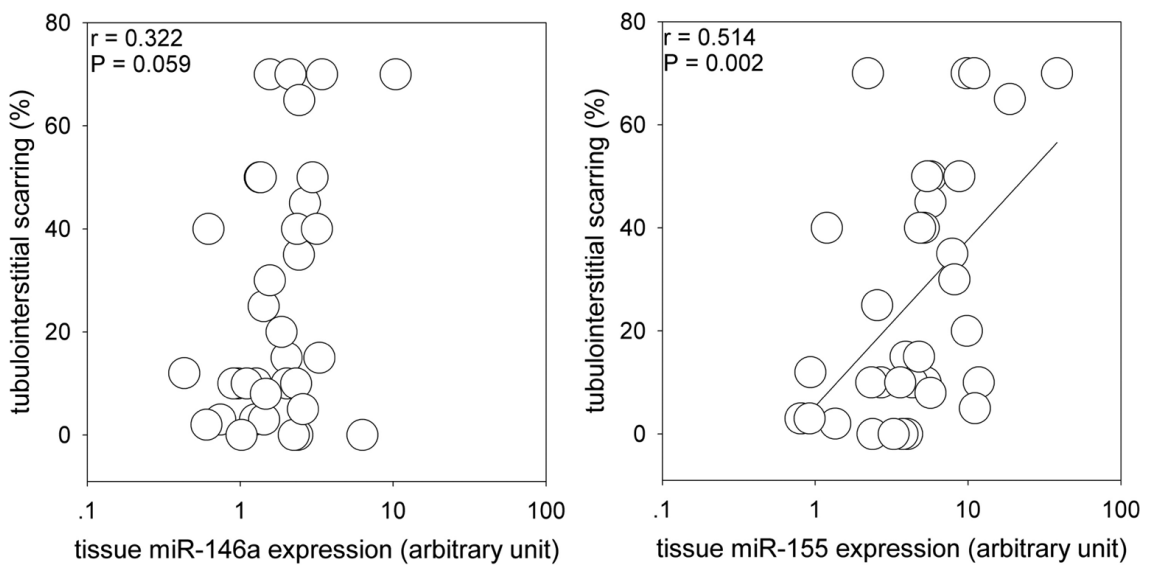

Fig. 5. Relation between intra-renal level of miRNAs and histological damage. Data are compared by Spearman's rank correlation coefficient.

positively correlates with urinary expression of FOXP3 and RANTES (Fig. 6B).

\section{Discussion}

Multiple miRNAs have emerged as important players in the pathogenesis of a variety of kidney diseases in recent years [22,23]. For instance, miR-15a and miR-17 are linked to polycystic kidney disease [24,25] and miR-21, miR-192, miR-216, miR-377 are linked to diabetic nephropathy [26-29]. Our previous study showed that intra-renal levels of miR-200c, miR-141, miR-205 and miR-192 was diversely regulated and correlated with disease severity and progression in patients with IgAN [15]. In the present work, we further proved up-regulation of both intra-renal and urinary levels of miR-146a and miR-155 and their correlations with clinical parameters and histological dam- age in patients with IgAN, suggesting participation of these two miRNAs in the pathophysiology of IgAN. It should be mentioned that intra-renal levels of these two miRNAs was detected in a previous study [30] but not another that investigated the expression profile of renal miRNAs in IgAN [14]. This discrepancy might be a result of different technology or materials used in these studies.

It is interesting to note that our previous study showed a reduced level of urinary miR-200a, miR-200b and miR-429 (all EMT-related miRNA) in IgAN [16], while we observed an increased in urinary miR-146a and miR-155 levels in the present study. Our result strongly suggests that the change in urinary miRNA levels represent specific alteration for individual miRNA target rather than being a generic phenomenon of all miRNA.

verwhelming evidence has proved the involvement of miR-146a and miR-155 in the regulation of both innate 

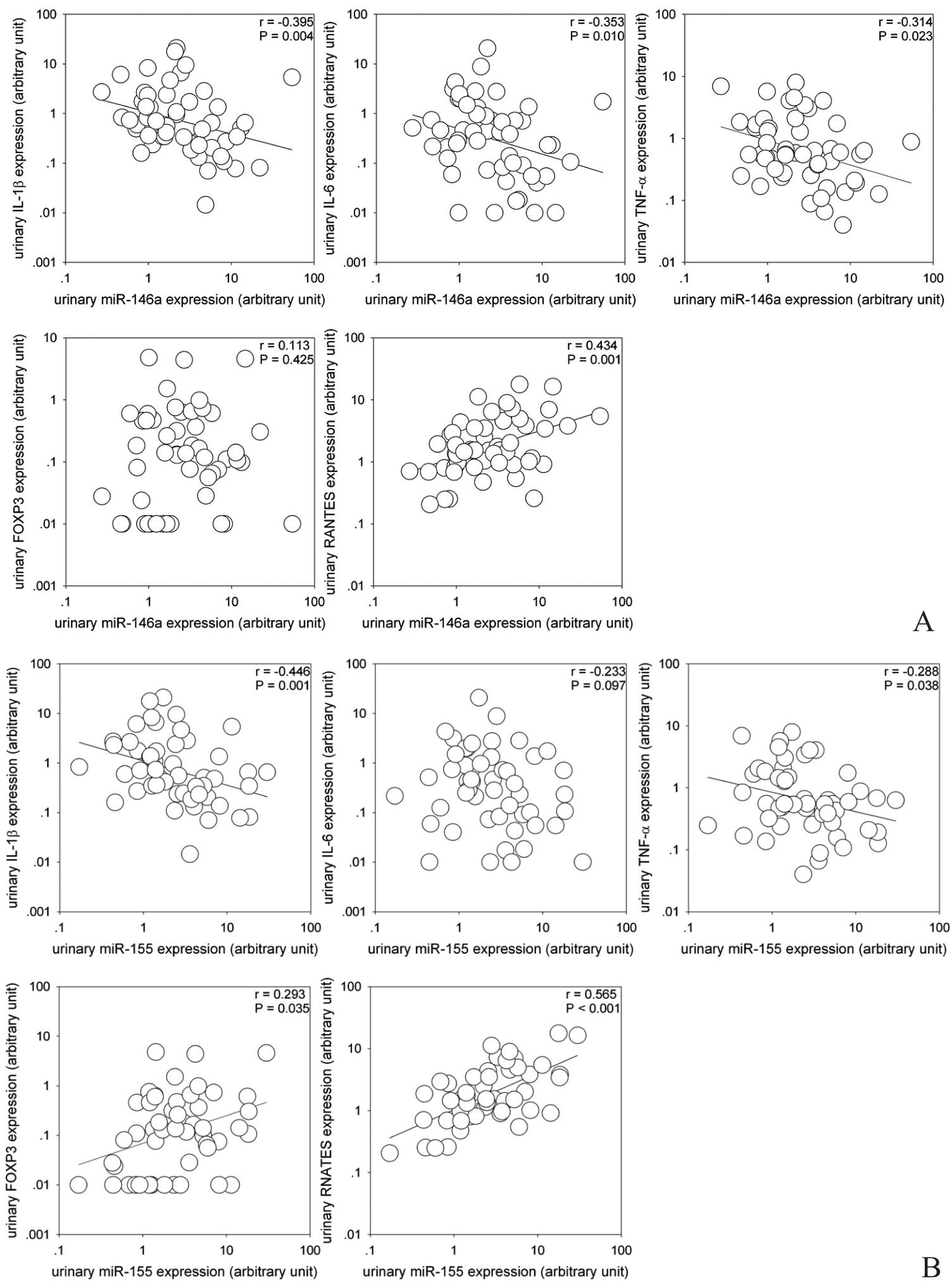

A 
and adaptive immune response [9-13]. Given the importance of renal immune response in the pathophysiology of IgAN, we investigated the correlations between these two miRNAs and important pro-inflammatory cytokines: IL-1- $\beta$, IL-6, and TNF- $\alpha$, a chemokine (i.e. RANTES), together with the regulatory $\mathrm{T}$ cell (Treg) marker FOXP3, in the urine sediment. In line with previous studies $[31,32]$ which showed that both miR$146 \mathrm{a}$ and $\mathrm{miR}-155$ exerted a regulation activity to limit over-production of pro-inflammatory cytokines, we found urinary levels of miR-146a and miR-155 negatively correlated with the expression of IL- $1 \beta$, IL- 6 and TNF- $\alpha$ in patients with IgAN. Our result supports the hypothesis that upregulation of $\mathrm{miR}-146 \mathrm{a}$ and $\mathrm{miR}-155$ results in suppression of IL- $1 \beta$ and IL- 6 production.

On the contrary, we found that urinary levels of miR$146 a$ and miR-155 positively correlated with RANTES. Our result is distinct different from previous studies, which showed that increased miRNA-146a level negatively regulates the release of RANTES in human lung alveolar epithelial cells and macrophages [31,33]. The effect of miR-146a and miR-155 on RANTES expression and function in IgAN requires further study.

We also found urinary level of miR-155 positively correlated with FOXP3. Treg cell has been suggested as an important player in IgAN and a numerical and/or functional deficit of Treg cell is hypothesized to trigger the development of the disease [34]. Recent studies proved that FOXP3 induced miR-155 production contributed to Treg development $[35,36]$. Our result supports these previous studies.

OThere are a few limitations of our study. First, we detected the levels of the studied miRNAs using whole renal tissue and urine sediment without determining the cellular sources for each of them. Although both miRNAs we examined in this study are related to immune response, they may be expressed in other cell types [33]. Future studies would be necessary to investigate miRNA level in specific renal cell type. Second, we did not conduct a function study of these miRNAs. The underlying mechanism of the changes and correlations observed in this study needs further investigation. Because of the limited among of RNA obtained from the kidney tissue and urine sediment, we did not quantify the expression level of other known targets, such as tumor necrosis factor receptor associated factor- 6 (TRAF6) and interleukin-1 receptor-associated kinase 1 (IRAK1), and compared them to intra-renal or urinary miRNA level. Finally, the present study is only cross-sectional in design, and it is possible that miRNA levels alter with disease progression and therapy. Fu- ture studies are needed to investigate the serial change of miRNAs level in IgAN.

In summary, we found both intra-renal and urinary levels of miR-146a and miR-155 are upregulated in IgAN, and the degree of up-regulation correlated with the disease severity. Urinary levels of miR-146a and miR-155 correlated with the expression of various inflammatory cytokines. Our results suggested miR-146a and miR-155 might play an important role in the pathophysiology of IgAN.

\section{Acknowledgement}

This study was supported in part by the CUHK research accounts 6901031 and 7101215. T he results presented in this paper have not been published previously in whole or part, except in abstract format. All authors declare no conflict of interest.

\section{References}

[1] J.V. Donadio and J.P. Grande, IgA nephropathy, $N$ Engl J Med 347 (2002), 738-748.

[2] F.C. Berthoux, H. Mohey and A. Afiani, Natural history of primary IgA nephropathy, Semin Nephrol 28 (2008), 4-9.

[3] C.H. Tan, P.T. Loh, W.S. Yang and C.M. Chan, Mycophenolate mofetil in the treatment of $\operatorname{IgA}$ nephropathy: a systematic review, Singapore Med J 49 (2008), 780-785.

[4] M.E. Lamm and S.N. Emancipator, The mucosal immune system and IgA nephropathy, Semin Nephrol 7 (1987), 280-282.

[5] F.B. Waldo, Systemic immune response after mucosal immunization in patients with IgA nephropathy, J Clin Immunol 12 (1992), 21-26.

[6] S.N. Emancipator, Immunoregulatory factors in the pathogenesis of IgA nephropathy, Kidney Int 38 (1990), 1216-1229.

[7] J. Feehally and A. Allen, Abnormalities of IgA1 production in IgA nephropathy, Nephrology 7 (2002), S100-S105.

[8] W.P. Kloosterman and R.H. Plasterk, The diverse functions of microRNAs in animal development and disease, Dev Cell 11 (2006), 441-450.

[9] K.M. Pauley, S. Cha and E.K. Chan, MicroRNA in autoimmunity and autoimmune diseases, J Autoimmun 32 (2009), 189-194.

[10] K.M. Pauley and E.K. Chan, MicroRNAs and their emerging roles in immunology, Ann N Y Acad Sci 1143 (2008), 226-239.

[11] Y. Bi, G. Liu and R. Yang, MicroRNAs: novel regulators during the immune response, J Cell Physiol 218 (2009), 467472.

[12] A.E. Williams, M.M. Perry, S.A. Moschos, H.M. LarnerSvensson and M.A. Lindsay, Role of miRNA-146a in the regulation of the innate immune response and cancer, Biochem Soc Trans 36 (2008), 1211-1215.

[13] I. Faraoni, F.R. Antonetti, J. Cardone and E. Bonmassar, miR155 gene: a typical multifunctional microRNA, Biochim Biophys Acta 1792 (2009), 497-505. 
[14] Y. Dai, W. Sui, H. Lan, Q. Yan, H. Huang and Y. Huang, Microarray analysis of micro-ribonucleic acid expression in primary immunoglobulin A nephropathy, Saudi Med J 29 (2008), 1388-1393.

[15] W. Gang, B.C. Kwan, F.M. Lai, P.C. Choi, K.M. Chow, P.K. $\mathrm{Li}$ and C.C. Szeto, Intra-renal expression of microRNAs in patients with IgA nephropathy, Lab Invest 90 (2010), 98-103.

[16] G. Wang, B.C. Kwan, F.M. Lai, K.M. Chow, P.K. Li and C.C. Szeto, Expression of microRNAs in the urinary sediment of patients with IgA nephropathy, Dis Marker 28 (2010), 79-86.

[17] A.S. Levey, J.P. Bosch, J.B. Lewis, T. Greene, N. Rogers and D. Roth, A more accurate method to estimate glomerular filtration rate from serum creatinine: a new prediction equation. Modification of Diet in Renal Disease Study Group, Ann Intern Med 130 (1999), 461-470.

[18] W. Mitch, Measuring the rate of progression of renal insufficiency, in: WE M, J S, BM B, ed., The Progressive Nature of Renal Disease, New York: Churchill Livingstone Inc (1986), pp. 203-320.

[19] S. Bhandari, N. Watson, E. Long, S. Sharpe, W. Zhong, S.Z. $\mathrm{Xu}$ and S.L. Atkin, Expression of somatostatin and somatostatin receptor subtypes 1-5 in human normal and diseased kidney, J Histochem Cytochem 56 (2008), 733-743.

[20] A. Biosystems, Endogenous Controls for Real-Time Quantitation of miRNA Using TaqMan ${ }^{\circledR}$ MicroRNA Assays, Application Note TaqMan ${ }^{\circledR}$ MicroRNA Assays (2007).

[21] D.R. Helsel, Less than obvious - statistical treatment of data below the detection limit, Environ Sci Technol 24 (1990), 1766-1774.

[22] M. Kato, L. Arce and R. Natarajan, MicroRNAs and their role in progressive kidney diseases, Clin J Am Soc Nephrol 4 (2009), 1255-1266.

[23] S. Saal and S.J. Harvey, MicroRNAs and the kidney: coming of age, Curr Opin Nephrol Hypertens 18 (2009), 317-323.

[24] S.O. Lee, T. Masyuk, P. Splinter, J.M. Banales, A. Masyuk, A. Stroope and N. Larusso, MicroRNA15a modulates expression of the cell-cycle regulator $\mathrm{Cdc} 25 \mathrm{~A}$ and affects hepatic cystogenesis in a rat model of polycystic kidney disease, $J$ Clin Invest 118 (2008), 3714-3724.

[25] H. Sun, Q.W. Li, X.Y. Lv, J.Z. Ai, Q.T. Yang, J.J. Duan, G.H. Bian, Y. Xiao, Y.D. Wang, Z. Zhang, Y.H. Liu, R.Z. Tan, Y. Yang, Y.Q. Wei and Q. Zhou, MicroRNA-17 posttranscriptionally regulates polycystic kidney disease-2 gene and promotes cell proliferation, Mol Biol Rep (2009), Oct 10.

[26] Z. Zhang, H. Peng, J. Chen, X. Chen, F. Han, X. Xu, X.
He and N. Yan, MicroRNA-21 protects from mesangial cell proliferation induced by diabetic nephropathy in $\mathrm{db} / \mathrm{db}$ mice, FEBS Lett 583 (2009), 2009-2014.

[27] M. Kato, J. Zhang, M. Wang, L. Lanting, H. Yuan, J.J. Rossi and R. Natarajan, MicroRNA-192 in diabetic kidney glomeruli and its function in TGF-beta-induced collagen expression via inhibition of E-box repressors, Proc Natl Acad Sci U S A 104 (2007), 3432-3437.

[28] Q. Wang, Y. Wang, A.W. Minto, J. Wang, Q. Shi, X. Li and R.J. Quigg, MicroRNA-377 is up-regulated and can lead to increased fibronectin production in diabetic nephropathy, FASEB J 22 (2008), 4126-4135.

[29] M. Kato, S. Putta, M. Wang, H. Yuan, L. Lanting, I. Nair, A. Gunn, Y. Nakagawa, H. Shimano, I. Todorov, J.J. Rossi and R. Natarajan, TGF-beta activates Akt kinase through a microRNA-dependent amplifying circuit targeting PTEN, Nat Cell Biol 11 (2009), 881-889.

[30] Y. Liang, D. Ridzon, L. Wong and C. Chen, Characterization of microRNA expression profiles in normal human tissues, BMC Genomics 8 (2007), 166.

[31] J. Hou, P. Wang, L. Lin, X. Liu, F. Ma, H. An, Z. Wang and X. Cao, MicroRNA-146a feedback inhibits RIG-I-dependent Type I IFN production in macrophages by targeting TRAF6, IRAK1, and IRAK2, J Immunol 183 (2009), 2150-2158.

[32] M. Ceppi, P.M. Pereira, I. Dunand-Sauthier, E. Barras, W. Reith, M.A. Santos and P. Pierre, MicroRNA-155 modulates the interleukin-1 signaling pathway in activated human monocytederived dendritic cells, Proc Natl Acad Sci U S A 106 (2009), 2735-2740.

[33] M.M. Perry, S.A. Moschos, A.E. Williams, N.J. Shepherd, H.M. Larner-Svensson and M.A. Lindsay, Rapid changes in microRNA-146a expression negatively regulate the IL-1betainduced inflammatory response in human lung alveolar epithelial cells, J Immunol 180 (2008), 5689-5698.

[34] H. Huang, Y. Peng, F. Liu and H. Lei, Is IgA nephropathy induced by abnormalities of CD4+CD25+Treg cells in the tonsils? Med Hypotheses 69 (2007), 410-413.

[35] Y. Zheng, S.Z. Josefowicz, A.Kas, T.T. Chu, M.A. Gavin and A.Y. Rudensky, Genome-wide analysis of Foxp3 target genes in developing and mature regulatory T cells, Nature $\mathbf{4 4 5}$ (2007), 936-940.

[36] S. Kohlhaas, O.A. Garden, C. Scudamore, M. Turner, K. Okkenhaug and E. Vigorito, Cutting edge: the Foxp3 target miR-155 contributes to the development of regulatory T cells, J Immunol 182 (2009), 2578-2582. 


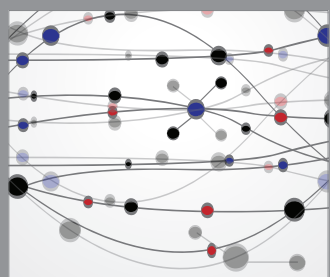

The Scientific World Journal
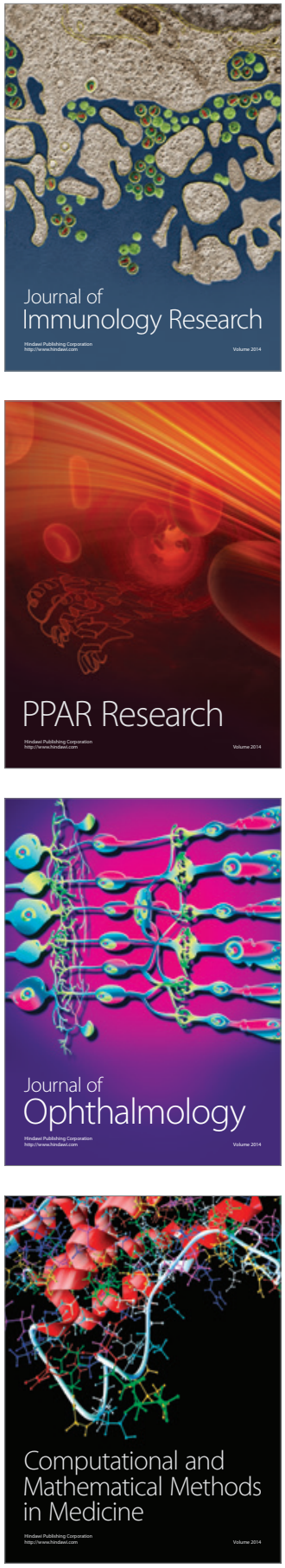

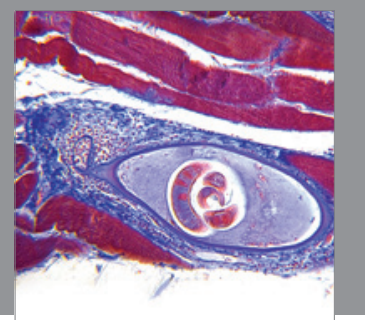

Gastroenterology

Research and Practice
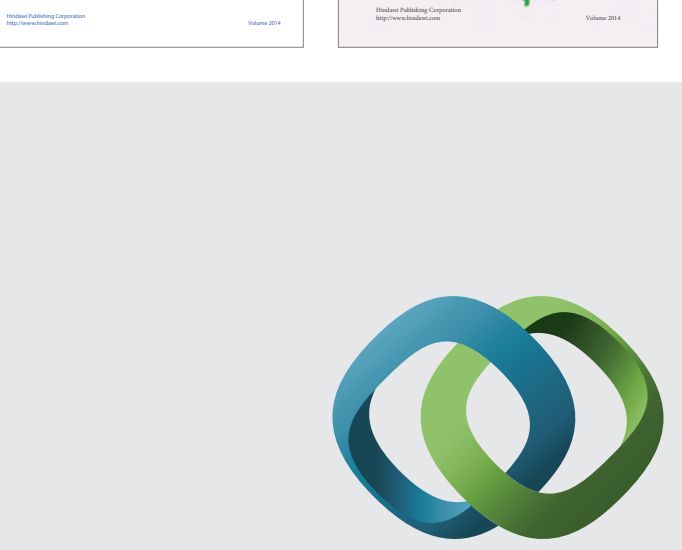

\section{Hindawi}

Submit your manuscripts at

http://www.hindawi.com
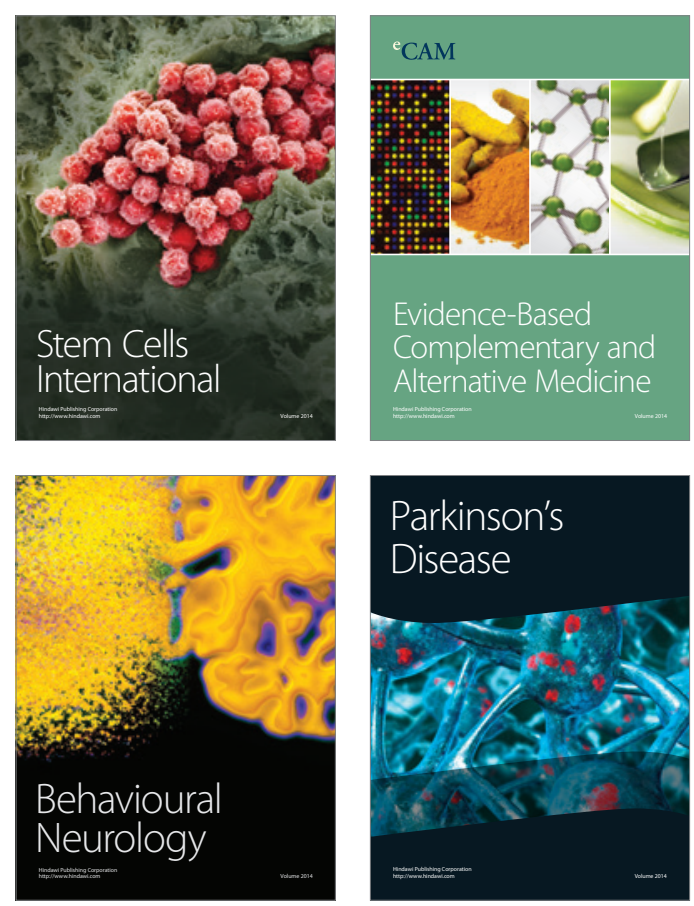

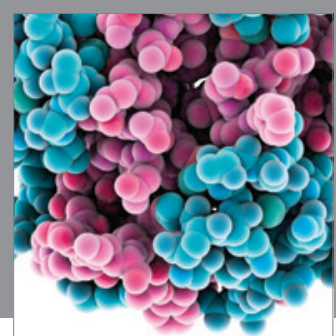

Journal of
Diabetes Research

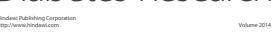

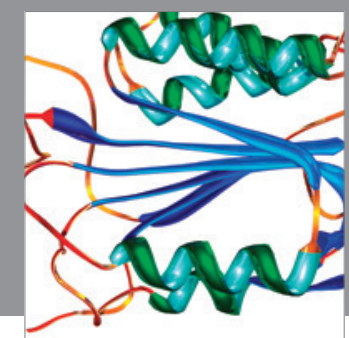

Disease Markers
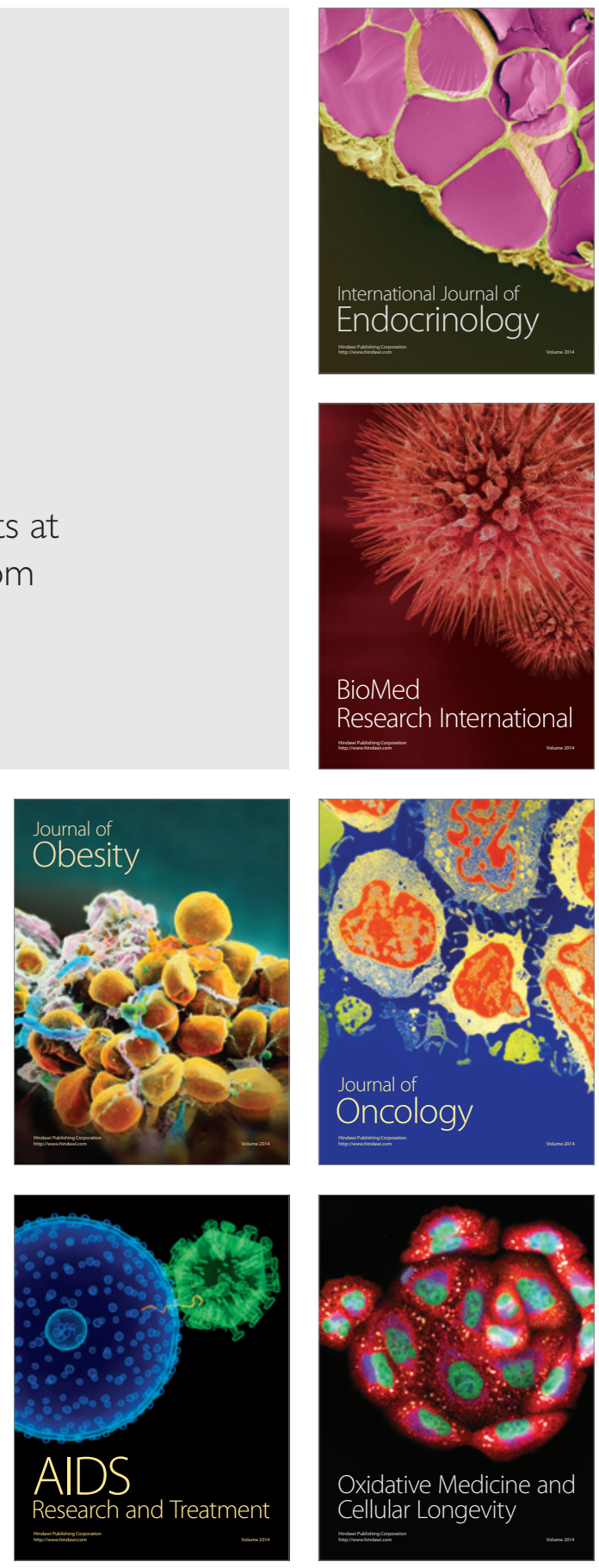